\title{
Microstructure Effect on LaPtBi Superconductivity
}

Cigdem Ozsoy-Keskinbora ${ }^{1}$, Yunbo $\mathrm{Ou}^{2}$, Debaleena Nandi ${ }^{2}$, Katie Huang ${ }^{3}$, Stephan Kraemer ${ }^{4}$, Philip $\mathrm{Kim}^{3}$, Amir Yacoby ${ }^{3}$, Jagadeesh Moodera ${ }^{5}$, David Bell ${ }^{1}$

${ }^{1}$ Harvard John A. Paulson School of Engineering and Applied Sciences, Harvard University

${ }^{2}$ Plasma Science and Fusion Center and Francis Bitter Magnet Laboratory, Massachusetts Institute of Technology)

${ }^{3}$ Department of Physics, Harvard University)

${ }^{4}$ Center for Nanoscale Systems, Harvard University)

${ }^{5}$ Department of Physics, Massachusetts Institute of Technology)

Recent studies predict that LaPtBi is a half-Heusler has multi-functionalities: the superconductivity ${ }^{1}$ and topological edge states, a namely topological superconductor under certain conditions ${ }^{2}$. It can be stimulated under substantial uniaxial strain conditions. The superconductivity in LaPtBi has been shown in bulk. However, the topological superconductive surface state can not be observed yet. In this study, we report the transmission electron microscopy work for observation of superconductivity and its relationship with a uniaxial strain in MBE grown epitaxial non-centrosymmetric LaPtBi film on MgO (001).

MEB growth parameters optimized to grow a compressive uniaxial strain of $17 \%$. In such a strained LaPtBi film, the predicted topological non-trivial gap at the $\Gamma$ point is expected to emerge. It's very challenging to measure the strain in such a thin film. Since the phenomena appear at very restricted and dedicated conditions, the characterization method must have very high sensitivity, resolution, localization, and precision. Transmission electron microscopy is a powerful technique to investigate structural, compositional or electromagnetic properties of materials. TEM also allows measuring strain spatial resolution in the range of nanometers for characterization of superconductor and topological materials, where small compositional variations have significant effects on the material properties.

In this work we will summarize the HRTEM, EDX, STEM studies had been carried out to understand the strain and wide range of superconductor transition temperature on MBE growth LaPtBi.

JEOL ARM 200F STEM equipped with a cold field-emission electron source, a probe Cs-corrector (CEOS $\mathrm{GmbH}$ ), Bruker windowless energy-dispersive X-ray spectroscopy (EDXS) detector, was used for investigations. In the light of HRTEM and STEM images, instead of epitaxial growth, unexpectedly columnar growth that is perpendicular to the growth direction. Another interesting observation was inhomogeneities in the thin film composition shown in figure 1. Thin film columns indicate different compositions of La, Pt and Bi atom.

\section{References}

1) O. Pavlosiuk, et. al. Scientific Reports, 6, 18797 (2016)

2) D. Xiao et al., Phys. Rev. Lett.105, 25 (2010).

3) This work was performed at the Center for Nanoscale Systems (CNS), a member of the National Nanotechnology Coordinated Infrastructure Network (NNCI), which is supported by the National Science Foundation under NSF award no. 1541959. 

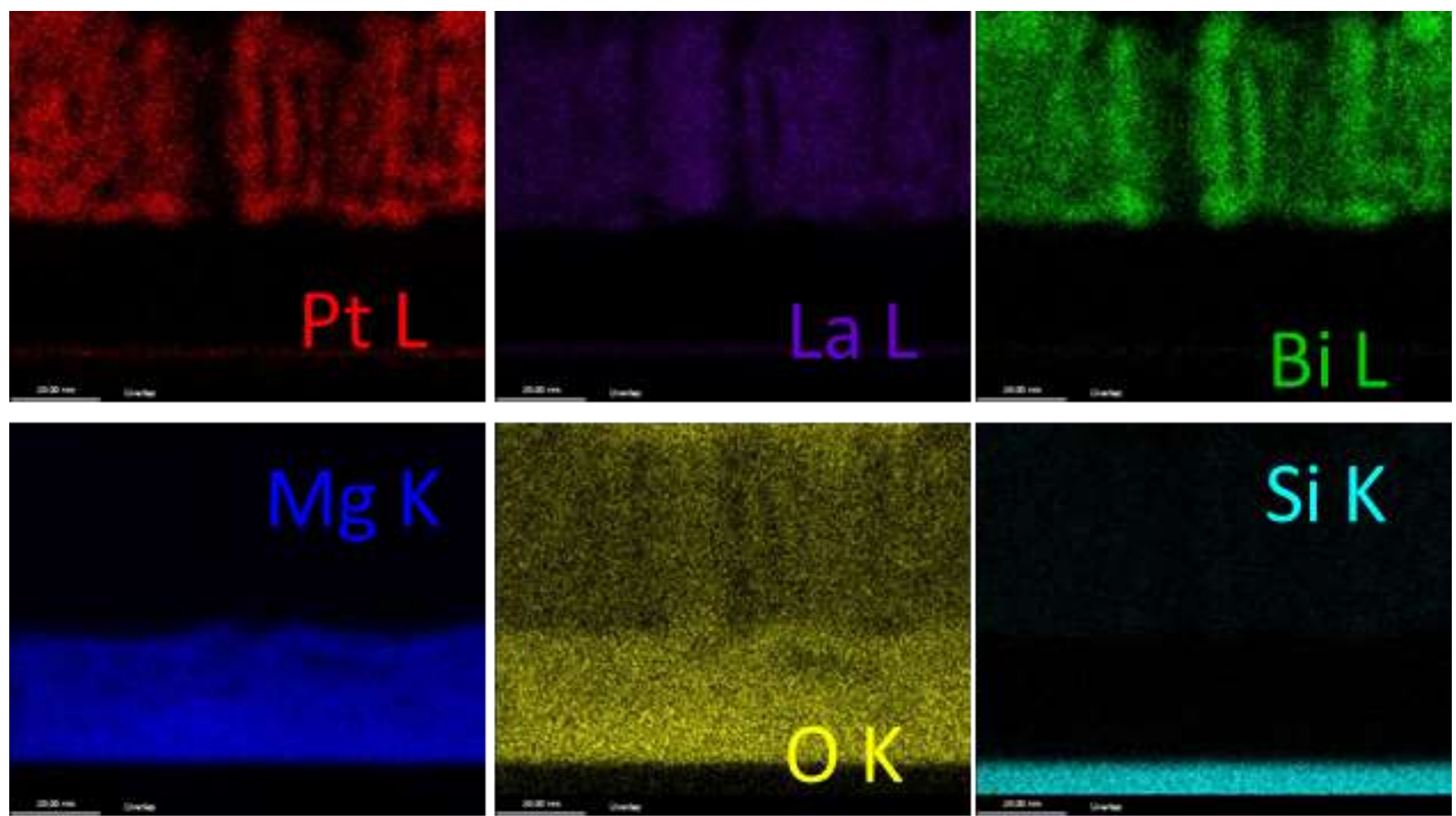

\section{Si K}

Figure 1. EDX elemental map of focused ion beam structured LaPtBi 